\title{
Bemerkungen über den Zusammenhang der Flächen.
}

\author{
Von Feuix Klein in Eriangex.
}

Die auf den Zusammenhang der Flächen bezüglichen Definitionen werden bei Riemann zumächst ohne besondere Festsetmngen hinsichtlich des Unendlich-Weiten hingestellt. Aber entsprechend der von ihm beabsichtigten Verwerthung dieses geometrischen Begriffes füx functionentheoretische Untersuchungen wird bei ihm eine solche Festsetzung implicite eingeführt, indem nämlich die unbegrenzte Ebene einer Kugelfäche äquivalent gesetzt wird, auf die sie durch stereographische Projection bezogen ist. In ähnlicher Weise kann man jede sich in's Unendliche erstreckende Fläche auf eine durchaus im Endlichen gelegene reduciren: man braucht sie nur einer Transformation durch reciproke Radien zu unterwerfen, dexen Inversionscentrum nicht selbst der Flüche angehört. Man kann dann alle Betrachtungen äber den Zusammenhang der Flächen, wie sie gewöhnlich unter der stillschweigenden Voraussetzung durchaus im Endhchen gelegeper Flächen angestellt werden, auf beliebig sich in's Unendliche erstreckende übertragen.

Aber die so eingeführte Festsetzung, vermöge deren das UnendlichWeite als ein einzelner Punkt erscheint*), ist an und für sich willkührlich; sie widerspricht überdies dem Wesen der Sache, wenn man, wie in der projectivischen Geometrie, das Unendlich-Ferne als eine zweifach ansgedehnte Mannigfaltigkeit von Punkten, als eine Ebene, betrachten muss. Auch bei dieser projectivischen Anschanungsweise kann man für Flächen, die sich beliebig in's Unendliche erstrecken, dieselben Probleme aufstellep, auf welche sich, bei durchaus im Endlichen gelegenen Flächen, die Riemann'schen Betrachtungen beziehen. Es fragt sich, ob dann specifisch neue Ueberlegungen noth-

*) Dieselbe ist auch in anderen auf die Analysis situs bezüglichen Fragen gelegentlich gebraucht worden; $\nabla g l$. z. B. Listing: Der Census der räumlichen Complexe, Góttinger Abhandlungen 1861. - Dass man und wie man dieselbe verwerthen kann, um anf sie ein ganzes" System der Geometrie zu begründen, welches als eine Art von Seitenstuck zur projectivischen Geometrie betrachtet werden darf, vgl. meine Sehrift: Vergleichende Betrachtungen üter newere geometrische Forschungen. Erlangen 1872. 
wendig werden, oder ob es gelingt, die betr. Probleme durch blosse Benutzung der Riemann'schen Betrachtungen zu erledigen. So ungefähr stellte ich diese Frage in einem neuerdings in diesen Annalen erschienenen Aufsatze (Ueber Flächen dritter Ordnung. t. VI.) ${ }^{*}$ ), ohne aber eine Beantwortung derselben in definitiver Form zu versuchen. Vielmehr machte ich nur anf eine Reihe von Schwierigkeiten aufmerksam, die sich einer unmittelbaren Verwerthung der Riemann'schen Betrachtungen entgegenstellen. Ich betonte besonders, dass der ungewöhnliche Zusammeuhang der unbegränzten Ebene in Anlehnung an die projectivische Anschauung nieht, wie in der Riem an n'schen Theorie, gleich Null, sondern gleich Z $Z$ wei zu setzen ist, falls man ohne nähere Festsetzung die gewö̈hnliche Definition dieses Zusammenhang's ${ }^{* *}$ ) beibehalten will. Denn die Ebene wird entsprechend der projectivischen Anschauung durch eine in ihr verlaufende Gerade, die doch auch eine geschlossene Curve ist, noch nicht in zwei Stücke getheilt, Dementsprechend glaubte ich die Zahlen, welche Hr. Schläfli in einem kurz zuvor veröffentlichten Aufsatze (Annali di Matemetica. t. V. Quand'è che dalla superficie generale di terz'ordine si stacca una parte etc.) für die fünf verschiedenen Arten der allgemeinen Fläehè dritter Ordnung aufgestellt hatte, alle um zwei Einheiten erhöhen zu sollen.

Ich bin nun von Hrn. Sehläfli brieflich darauf aufmerksam gemacht worden, dass man, unbesehadet der Richtigkeit dieser meiner Betrachtungen und Einwände, doch auch bei projectivischer Anschaung für die unbegränzte Ebene den Zusammenhang Null ansetzen kann, wenn man dieselbe nämlich als Doppelflä che betrachten will, also etwa als Grïnze eines zweischaligen Hyperboloid's. In der That, man denke sich, um eine bestimmte Vorstellung zu haben, tine Ebene horizontal und ziehe in ihr eine Linie Süd-Nord. Dann wird die als Doppelflïche betrachtete Ebene in zwei Theile zerfallen, deren einer die östliche Hälfte des oberen Blattes und die westliche des unteren, deren

*) Dass sich die Problemstellung der Analysis situs je nack der Beurtheilung des Unendlich-Weiten modificirt, hatte ich bereits in der oben citirten Schrift: "Vergleichende Betrachtungen etc." angegeben ( $p$. 30 derselben).

**) Wenn man auf eioer geschlossenen Fläche im Maximum $q$ geschlossene Curven ziehen kann, ohne die Fläche zu zerstücken, so setzt Riemann den Zusammenhang derselben $=2 q+1$. Aber es ist bereits in den Neumann'schen "Vorlesungen über Riemann's Theorie ete." argedeutet und neuerdings von Hrn. Schläfli hervorgehoben worden (vgl. z. B. Borchardt's Journal. to 76. p. 152. Note), dass es consequenter ist, in einem solchen Falle nur von einem $2 q$-fachen Zusammenhange zu sprechen. Indem ich mich im Texte dieser Bezeichnung anschliesse, füge ich, nach dem Vorgange Schläfli's, wo eine Undeutlichkeit entstehen könnte, dem Worte "Zusammenhang" das Attribut "ungewöhnlich" hinzo. 
anderer die beiden übrigen Hälften enthält. Dabei wird die gerade Linie selbst doppelt gedacht, nämlich sowohl im oberen als in unteren Blatte verlaufend.

Eine solche Einführung von Doppelfächen erscheint um so mehr zulässig, als sie schon in den Untersuchungen der Analysis situs, welche sich nur auf im Endlichen gelegene Flächen beziehen, nothwendig wird*). Ein bekanntes Beispiel dafür bietet die (mit einer Randcurve versehene) Fläche, welche mąn aus einem Papierstreifen bilden kann, indem man die beiden Enden des Streifens so aneinanderheftet, dass die eine Seite desselben in die andere übergeht. Ein anderes Beispiel für eine solche Fläche, und zwar eine gesehlossene Hläche, giebt, wie weiter unten gezeigt werden soll, die Steiner'sche Fläche, die man ja bekanntlich als völlig im Endlichen gelegen voraussetzen darf. (Von den isolirten Stücken, welche ihre Doppelgeraden besitzen, wird dabei abgesehen.)

Ich habe nun gefunden, dass man dịe Theorie des Flächenzusammenhangs, wie sie gewöbnlich entwickelk wird, in der That auf die projectivischen Vorstellungen unverändert übertragen kann, wenn man sich überhaupt entschliesst, die unpaaren Flächen der projectivischen Geometrie als Doppelflächen zu betrachten, und eine unpaare Curve erst dann als geschlossen anzusehen, wenn man sie zweinal durchlaufen hat.

$\mathrm{Ob}$ man sich dieser Anschauung anschliessen will, oder nicht, wird zunächst dem freien Entschlusse des Eipzelnen überlassen sein. Es mag sogar hervorgehoben werden, dass es von vorneherein sehr unnatürlich scheint, in der projectivischen Geometrie die Ebene als Grenzfall der nicht-geradlinigen Flächen zweiter Ordnung betrachten zu sollen. Aber die Anschaung empfiehlt sich durch ihren Erfolg. Denn man kann im Anschlusse an sie auch für die projectivische Auffassung des Unendlich-Weiten den Satz aussprechen, der den Zusammenhangsbegriff für die functionentheoretischen Untersuchungen so werthvoll macht: dass nämlìch zwei geschlossene Flächen dann und nur dann auf einander Punlt für Punkt bezogen werden können, so dass consecutiven Punkten der einen consecutive Punkte der anderen entsprechen, wenn der Zusammenhang der beiden Flächen der gleiche ist. (Für ungeschlossene Flächen kommt nur die weitere Bedingung hinzu,

*) Freilich verlangt dann die Consequenz, dass man, wenn von einer fläche, deren entgegengesetzte Seiten richt in einander uborgehen, schlechthin die Rede ist, nuter dem Zusammenhange der Fläche die Grundzahl versteht, die man dem von den beiden Seiten gebildeten Systeme beizulegen hat (cf. Neumann"s Vorlesungen). Der Zusammenhang der Kugel wäre dann $=-2$; der Zusammenhang der einzelnen Kugelseite $=0$. 
dass auch die Anzahl der Randeurven bei beiden übereinstimmen muss*)\}.

Dem widerspricht nicht, wie vorab bemerkt sei, wenn ich in meiner schon genannten Arbeit hervorhob, das einschalige Hyperboloid und die Ringfiäche seien nicht in einander überführbar, obgleich ihr Zusammenhang nach S chläfli's wie nach meiner Zählung übereinstimmend gleich Zwei zu setzen ist. Denn unter Ueberführbarkeit wurde damals etwas Anderes verstanden, als hier für die Transformirbarkeit zweier Flächen in einander verlangt wird. Eine Fläche wurde damals (vol. § 16. meiner Arbeit) in eine andere überführbar genannt, wenn es gelang, durch Verbindung von Collineationen, die das Unendiche in's Endliche bringen, mit stetigen, unendlich kleinen Transformationen, die nur das Findliche betreffen, die eine Fläche aus der anderen abzuleiten. Durch solche Mittel ist das einschalige Hyperboloid allerdings nicht in eire Ringfläche zu verwandeln.

Dagegen kann eine eindeutige, stetige Beziehung zwischen beiden durch den folgenden urstetigen Process ohne Weiteres hergestellt werden: Man zerschneide das Hyperboloid längs der unendlich fernen Ebene, bringe die so entstandene zweifach berandete Fläche durch Verzerrung ganz in's Endliche und hefte die beiden Ränder dann wieder in der Weise an einander, dass jeder Punkt des einen Randes mit demjenigen des anderen vereinigt wird, von dem er vorher durch den Schnitt getrennt worden war. (Damit dies geschieht, muss man den einen Rand des Hyperboloids gegen den anderen Rand um $180^{\circ}$ drehen, ehe man die Ränder durch Zusammenbiegen vereinigt). - Aehnliche unstetige Processe zur Herstellung der eindeutigen Beziehung werden übrigens schon nothwendig, wenn man nur von Flachen handelt, die ganz im Endlichen liegen. Man kann z. B. eine einfach berandete, mit einem Verzweigungspunkte versehene Riemann'sche Fläche nur dadurch mit einem einfach zusammenhängenden Stücke einer Ebene zur Deckung bringen, dass man sic durch einen vom Verzweigungspunkte zum Rande gehenden Schnitt zerschneidet und hinterher die durch den Schnitt getrennten Partieen wieder an einander heftet. Ich finde, dass die Darstellungen dieser Theorie, welche mir gerade zur Hand sind, hierüber keine volle Klarheit geben: es wird von der Möglichkeit eines solehen Zersehneidens and Wieder-Verbindens gesprochen, aber dasselbe wird nur als vützlich, nicht als für viele Fälle nothwendig dargestellt. - Es ist wohl kaum nöthig, hervorzuheben, dass der Unterschied, den ich zwischen Hyperboloid und Ringfläche

*) Dass diese Bedingungen für die Transformirbarkeit zweier Flächen in der That hinreichend sind, findet sich kurz und übersichtlich bei C. Jordan bewiesen in Liouville's Journal t. XI. 1866. 
und überhaupt zwisehen paaren und unpaaren Flächen in meiner früheren Arbeit hervorhob, durch diese Bemerkungen nicht bedeutungslos wird; er kommt nur bei der Frage nach der Möglichkeit der eindeutigen Beziehnng zweier Flächen auf einander, wie sie hier gestellt wird, nicht in Betracht. -

Den Beweis für das aufgestellte Theorem erbringe ich in der Weise, dass ich den Raum mit unendlich ferner Ebene zu dem Raume mit unendlick fernem Punkte in eine eirfache ein-zweideutige Beziełung setze, vermöge deren das Theorem unmittelbar aus dem gleichlantenden Satze der gewöhnlichen Analysis situs hervorgeht.

Es mag das zunächst bei der Ebene erörtert werderi. In der gewöhnlichen Analysis situs wird die Ebene einer Kngel äquivalent gesetzt, auf die sie durch stereographische Projection bezogen ist. Aber man kann die Kugel dann weiterbin durch centrale Projection auf eine neue Ebene beziehen. Diese Ebene ist dann doppelt von den Bildern der Kugelpunkte tiberdeckt; ihre unendlieh ferne Gerade kommt als solche zur Geltung, indem ihr ein grösster Kreis auf der Kugel entspricht. Sieht man nun von der Kugel ab, welche die Beziehung zwischen den beiden Ebenen vermittelte, so hat man eine ein-zweideutige Verwaudtschaft 2 wischen der Fbene mit unendlich ferner Geraden und der Ebene mit unendlich fernem Punkte, vermöge deren jeder in der letzteren gezogenen Curve, falls man nur die unpaaren Curven der ersteren als Doppel-Curven ansieht, eine Curve der ersteren Ebene eindeutig entspricht.

Ganz entsprechend kaun man eine ein-zweidentige Beziehung zwischen den beiden Räumen herstellen. Da sich der Process bei ihnen, wegen des Nothwendigwerden's einer vierten Dimension, minder anschaulich bezeichnen lässt, so mögen die formeln hergesetzt werden, welche ihn repräsentireu. Sind $x, y, z$ rechtwinklige Coordinaten des Raumes mit unendlich fernem Punkte, $x^{\prime}, y^{\prime}, z^{\prime}$ des Raumes mit unendlich ferner Ebene, ist endlich $\lambda$ eine beliebige Constante, so hat man zu setzen:

$x^{\prime}=\frac{x}{1-2\left(x^{2}+y^{2}+z^{2}\right)}, \quad y^{\prime}=\frac{y}{1-2\left(x^{2}+y^{2}+z^{2}\right)}, \quad z^{\prime}=\frac{z}{1-\lambda\left(x^{2}+y^{2}+z^{2}\right)}$.

Jeder Fläche des Raumes mit unendlich fernem Punkte entspricht dann eindeutig eine Fläche des anderen Raumes, vorausgesetzt, dass man seine unpaaren Flächen als Doppelfächen betrachtet, und unser Theorem betr. die Flächen des letzteren Raumes ist richtig, weil es für die Flächen des anderen gitt.

Es mögen hier noch einige Bemerkungen Platz finden, die sich auf gewisse Beziehungen erstrecken, welche zwischen den Zusammenhange 
algebraischer Flächen und ihrem Verhalten bei algebraisch-eindeutigen Transformationen Statt finden.

Wenn zwei Flächen algebraisch eindeutig in einander übergeführt werden können, wenn dabei jedem reellen Punkte der einen ein reeller Punkt der anderen entspricht*), wenn endlich auf keiner von beiden dabei reelle Fundamentalpunkte auftreten, so haben die Flächen ersichtlich denselben Zusammenhang. Man kann von dieser Bemerkung Gebrauch machen, um den Zusammenhang gewisser algebraischer Flächen ohne Weiteres zu bestimmen. Die vierte der von Schlä̂lli aufgezählten Flächen dritter Ordnung $z$. B. (welche nor 3 reelle Gerade und 7 reelle Dreiecksebenen besitzt) lässt sich auf die Ebene ohne Zwischentreten reeller Fundamentalpunkte reell abbilden, denn die 6 hei ihrer Abbildung im algebraischen Sinne vorhandenen Fundamentalpunkte sind paarweise conjugirt imaginär. Der Zusammenhang der Fläche ist daher Noll; überdiess ist sie eine Doppelfäche, wejl jedem Punkte der Eberie, unabhängig davon, ob man ihn der einen oder anderen Seite der Ebene zurrechnet, ein und derselbe Punkt der Fläche entspricht. Aus demselben Grunde ist, wie bereits oben angegeben wurde, die Steiner'sche Fläche eine Doppelfäche vom Zusammenhange Null; denn es ist bekannt, dass sie ohne Auftreten von Fundamentalpunkten durch reelle Functionen zweiten Grades auf die Ebene abgebildet werden $\mathrm{kann}^{* *}$ ).

Es drängt sich nun ron selbst die Frage auf, welche Beziehungen für den Zusammenhang zweier Flächen gelten, die zwar auf reelle Weise algebraisch eindeutig auf einander bezogen werden können, bei deren Abbjldung aber reelle Fundamentalpunkte auftreten. Dabei müssen natürlich solche Flächen von der Betrachtung ausgeschlossen sein, die nicht isolirte, vielfache Punkte besitzen. Denn es wird bei den Definitionen des Zusammenhangs, wie sie gewöhnlich gegeben werden, überhaupt von solchen Flächen abgesehen. Lässt man sie bei Seite, so kann man folgenden Satz aussprechen: Finden sich auf der einen Fläche $\mu$, auf der anderm $\nu$ (reelle) Fundamentalpunkte, so ist der Zusammenhang der ersten, vermehrt um $\mu$, gleich dem Zusammenharg der zweiten, vermehrt um $v$.

*) Dies ist nicht immer der Fall, Z. B. kann die Fläche dritter Ordnung ohne Knoten, mit 3 reellen Geraden und 13 reellen Dreiecksebenen (die fünfte Art der allgemeinen Fläche nach Schläfli's Eintheilung) nar durch Functionen mit complexen Coefficieuten eindeutig auf die Ebene abgebildet werden.

*) Es sei hier daran erinnert, dass Clebsch gelegentlich seiner Untersuchung der Linienfäichen vom Geschlechte Null (Math. Annalen Bd. V.) alle Flächen, die ohne Auftreten ron (reellen oder imaginären) Fundamentalpunkten algebraiscb eindeutig auf einander bezogen werden können, als Flächen eines Typus zusammenfasst. 
Einige Beispiele mögen das zunächst erläutern.

1. Zwei Ebenen sollen algebraisch eindeutig (durch eine Cremon a-Transformation) auf einander bezogen sein. Dann ist bekannt, dass die Zahl der beiderseits überhaupt auftretenden (reellen und imaginären) Fundamentalpunkte die gleiche ist; gemäss unserer Behauptung muss auch die Zahl der reellen Fundamentalpunkte allein beiderseits übereinstimmen.

2. Eine Kugel werde stereographisch auf die Ebene (die Doppelebene) bezogen. Dann entspricht die Ebene eindeutig dem Aggregate der beiden Kugelflächen, welche durch die innere und äussere Seite der Kugel vorgestellt werden, und die zusammen nach einer bereits oben gemachten Bemerkung den Zusammenhang -2 repräsentiren. In der That treten anf der Kugel bei der Abbildung zwei Fundamentalpunkte auf, nämlich sowohl auf der änsseren als der inneren Seite je einer.

3. Ein einschaliges Hyperboloid werde durch stereographische Projection auf eine Ebene bezogen. Dann ist wiederum die eine Seite der Fläche der einen Seite der Ebene, die andere der zweiten Seite der Ebene entsprechend gesetzt, und man hat nicht sowohl ein einzelnes Hyperboloid, als ein System von zwei Hyperboloiden mit der Ebene zu vergleichen. Jedes der beiden Hyperboloide trägt einen Fundamentalpunkt: den Projectionspunkt. Aber die Ebene trägt vier Fundamentalpunkte; denn man muss die beiden Fundamentalpunkte, von denen man bei der Abbildung eines Hyperboloids gewöhnlich spricht, hier doppelt zählen, weil sie sowohl der einen als der anderen Seite der Ebene angehören. Der Zusammenhang des Hyperboloidsystems wird daher gleich +2 , wie es in Uebereinstimmung mit der gewöhnlichen Zählung sein muss; denn jedes der beiden Hyperboloide ist zweifach zusammenhängend, und ihre Trennung zählt für -2 .

4. Wenn man die drei ersten Schläfli'schen Arten der allgemeinen Fläche dritter Ordnung (die bez. 27, 15, 7 reelle Gerade enthalten) auf die Ebene abbildet, so erscheinen in der Ebene bez. $6,4,2$ reelle Fundamentalpunkte, die man wiederum doppelt $\mathrm{zu}$ zählen hat. Auf der Fläche selbst treten keine Fundamentalpankte auf. Dementsprechend wird der Zusammenhang bez. gleich 12, 8, 4. Schläfli gab die Zahlen 6,4,2. Aber er betrachtet bei seiner Abrählung die Flächen als einfache, nicht als Doppelflächen. Thut man das Letztere, so hat man die Processe, vermöge deren Schläfli diese Flächen aus der mit dem Zusammenhange Null vorausgesetzten, uubegrenzten Ebene herstellt, in der That doppèlt zu zählen, da sie sich auf beide Seiten der Fläche beziehen. Schläfli's Zählung war von dem hier entwickelten Standpunkte aus inconsequent, weil die 
Annahme, dass die Ebene nullfach zusammenhängend sei, bereits darüber entscheidet, dass man die Ebene als Doppelebene betraehten muss. Will man die Ebene und dementsprechend diese Flä́chen als einfache betrachten, so ergeben sich für die Flächen die Zusammenhangs-Zahlen 8,6, 4, wie ich in meiner früheren Arbeit ausführte. -

Um den Beweis der nunmebr an einzelnen Beispielen erläuterten Regel zu führen, ist es nöthig, für reelle Elemente insbesondere einige Ueberlegungen zu entwickeln, welche sonst nur für beliebig complexe Elemente in der Theorie des algebraisch-eindeutigen Entsprechens bewiesen werden.

Es seien zwei geschlossene Flächen auf einander reell eindeutig bezogen. Jedem Fundamentalpunkte der einen Fläche entspricht auf der anderen eine fundamentalcurve. Es ist leicht zu sehen, dass diese Curve nur aus einem Zuge bestehen kann. Denn man muss sich die Vorstellung bilden, dass dem Büschel von Fortschreitungsrichtungen, welche vou dem Fundamentalpunkte ausgehen, eindeutig die Punkte dieser Curve entsprechen: sowie jenes Büschel ohne Unterbrechung ist, so müssen die Punkte der Curve eine einzige, zusammenhängende Reihenfolge bilden. Die den verschiedenen Fundamentalpunkten der einen Fläche entsprechenden Fundamentalcurven werden sich, wie leicht zu sehen, nur in den Fundamentalpunkten der zweiten Fläche schneiden können. Sie werden durch sie in eine Anzahl, $S$, von Seg. menten zerlegt, die gleich ist der Anzahl von Malen, dass sie überhaupt durch die Fundamentalpunkte durchgehen.

Man überzeugt sich nun von folgendem Satze: Die Anzahl $S$ der Segmente, in welche die Fundamentalcureen zerlegt werden, ist auf beiden Flächen dieselbe. Geht nämlich die Fundamentalcurve, welche dem $i^{\text {ton }}$ Fundamentalpunkte der ersten Fläche entspricht, $a_{i k}$ mal durch den $k$-Fundamentalpunkt der zweiten, so wird auch die Curve, welche letzterem auf der ersten Fläche entspricht, $a_{i k}$ mal durch den $i^{\text {ten }}$ Fundamentalpunkt der ersten Fläche gehen müssen. Denn durch beide Behauptungen wird nur gleichmässig ausgesprochen, dass $a_{i k}$ Fortschreitungsrichtungen in dem einen und dem anderen Fundamentalpunkte existiren, welche einander entsprechen. Die Zahl $S$ ist aber die Summe aller $a_{i k}$; sie fällt also beiderseits gleich aus. - Durch die $a_{i k}$ Fortschreitungsrichtungen, welche den verschiedenen Fundamentalpunkten $k$ der zweiten Fläche entsprechen, wird das Büschel der von dem $i^{\text {ten }}$ Fundamentalpunkte der ersten Fläche ausgebenden Richtungen in $\sum_{k} a_{i k}$ Theile, oder, wenn man will, in $2 \sum_{k} a_{i k}$ Halbbüschel zerlegt. In eben soviele Segmente wird die dem $i^{\text {ten }}$ Fundamentalpunkte auf der zweiten Fläche entsprechende Curve durch die Fundamentalpunkte der zweiten Fläche getheilt; der 
Unterscheidung der Halbbüschel entspricht die Unterscheidung der beiden Seiten der verschiedenen Segmente.

Nunmebr wandele man jede der keiden Flächen in folgender Weise um. Man hebe die $\mu$ Fundamentalpankte der ersten Fläche und die $\nu$ Fundamentalpunkte der zweiten heraus (d. h. man schneide aus den Flächen kleine Stücke aus, welche diese Punkte umgeben). Dadurch wächst der Zusammenhang der ersten Fläche $\mu$ n $\mu$, der der zweiten um $v$. Sodann zerschneide man die Flächen längs der auf ihnen liegenden Fundamental-Curven: eine Operation, welche uun, nachdem dureh das Herausheben der Fundamentalpunlte Begränzungen entstanden sind, in dem Ziehen von $S$ Quersehnitten besteht. So ist die eine Fläche um $\mu-S$, die andere um $\nu-S$ im Zusammenhange vermehrt; die so veränderten Flächen sind aber nunmehr ohne Zwischentreten von Fundamentalpunkten eindeutig auf einander bezogen. Der Rand jeder der beiden so erhaltenen Flächen besteht, wie der grösseren Bestimmtheit wegen hervorgehoben sei, abwechselnd aus Segmenten von Fundamentalcurven und Stückeu der am Fundamentalpunkte herumgelegten kleinen Ovale, wie sie auf diesen Ovalen durch die von den Fundamentalpunkten ausgehenden Halb-Büschel bestimmt werden. Die beiden Ränder der beiden Flächen sind dann so eindeutig auf einander bezogen, dass jedem Stücke des einen, welches aus einem Segmente einer Fundamentalcurve besteht, ein Stück des anderen zugeordnet ist, welches einem der kleinen Ovale angehört, und umgekehrt. Zu jedem von einem Halbbüschel ausgeschnittenen Stück eines Ovals gehört ein zweites, welches durch das complementäre Halbbï:chel bestimmt wird. Dem entspricht, dass aueh jedes Segment einer Fundamentalcurve zweimal in der Begrenzung: auftritt. - Aber der Zusammenhang der beiden so erhaltenen Flächen ist um $\mu-\mathbf{S}$ und bez. $\nu-S$ grösser, als der Zusammenhang der beiden ursprünglichen klächen; addirt man also zum Zusammenhange der einen ursprünglichen Fläche $\mu$, zu dem der anderen $\nu$, so erhält man gleiche Zahlen, wie behauptet wurde.

Erlangen, Februar 1874. 